\title{
Longitudinal Changes in the European League Against Rheumatism Sjögren's Syndrome Patient Reported Index in Real-Life Practice
}

\author{
Ji Hyoun Kim ${ }^{1}$, You-Jung $\mathrm{Ha}^{2}$, Eun Ha Kang ${ }^{2}$, Yeong Wook Song ${ }^{3,4}$, Yun Jong Lee ${ }^{2,3}$ \\ Department of Internal Medicine, 'Chungbuk National University Hospital, Cheongju, ${ }^{2}$ Seoul National University Bundang Hospital, Seongnam, \\ ${ }^{3}$ Seoul National University College of Medicine, ${ }^{4}$ WCU Department of Molecular Medicine and Biopharmaceutical Sciences, Medical Research \\ Institute, Seoul National University College of Medicine, Seoul, Korea
}

\begin{abstract}
Objective. To investigate longitudinal changes in the European League Against Rheumatism (EULAR) Sjögren's syndrome patient reported index (ESSPRI) and to study the clinical features associated with favorable ESSPRI changes in primary Sjögren's syndrome (pSS). Methods. At baseline and after a median period of 6.6 years, 41 pSS patients were evaluated using the ESSPRI, EULAR Sjögren's syndrome disease activity index (ESSDAI), short-form 36, xerostomia inventory (XI), and visual analog scale (VAS) scores for symptoms. The favorable subgroup included patients who were stable or showed improved to satisfactory symptom status $($ ESSPRI $<5)$ and the unfavorable subgroup included those with stable or worsening to an unsatisfactory symptom status (ESSPRI $\geq 5)$. Results. Median ESSPRI increased from 4.11 to $5.33(p<0.05)$, although XI scores $(p=0.01)$ and oral dryness $(p<0.05)$ were significantly decreased. Serum immunoglobulin $\mathrm{G}$ level was significantly reduced $(p<0.001)$ but ESSDAI scores were unchanged. Six (14.6\%) patients showed clinical improvement in ESSDAI, and $11(26.8 \%)$ showed improvement in ESSPRI. On comparing the favorable $(n=17)$ and unfavorable $(n=24)$ subgroups, the former exhibited significantly lower VAS scores for sicca and depression and XI and ESSPRI scores at baseline (all $p<0.05)$ and more lacrimal flow $(p<0.05)$. The favorable subgroup received a significantly lower cumulative dose of pilocarpine and glucocorticoids (both $p<0.05$ ). Conclusion. About $25 \%$ of pSS patients showed clinically significant ESSPRI improvement and about $40 \%$ showed a favorable ESSPRI course. Because the favorable subgroup had more lacrimal flow and less sicca symptoms at baseline, long-term patient-derived outcomes could depend on residual exocrine function at pSS diagnosis. (J Rheum Dis 2019;26:191-199)
\end{abstract}

Key Words. Sjögren's syndrome, Patient outcome assessment, Quality of life, Xerostomia, Xerophthalmia

\section{INTRODUCTION}

Primary Sjögren's syndrome (pSS) is a chronic systemic autoimmune disease characterized by lymphocytic cell infiltration of salivary and lacrimal exocrine glands, resulting in functional impairment. However, the inflammatory process can extend beyond the exocrine glands and affect various non-exocrine organs such as the joints, skin, lungs, kidneys, nerves, and small vessels $[1,2]$. Although the prevalence or incidence of pSS is debated, it has been considered a rare disease and a recent meta-analysis reported a global worldwide prevalence of $0.06 \%[3,4]$.

A variety of extraglandular manifestations have been reported in pSS patients and a large Spanish registry revealed that $13 \%$ had a potentially life-threatening systemic manifestation [5]. However, most pSS patients have benign subjective conditions such as sicca symptoms, arthralgias, and fatigue. In line with these findings, pSS has not been associated with increased mortality [6,7], even though it negatively affects health-related quality of life [8-10].

Received : January 12, 2019, Revised : May 15, 2019, Accepted : May 28, 2019

Corresponding to : Yun Jong Lee (iD http://orcid.org/0000-0001-7615-8611

Department of Internal Medicine, Seoul National University Bundang Hospital, 82 Gumi-ro 173beon-gil, Bundang-gu, Seongnam 13620, Korea. E-mail : yn35@snu.ac.kr

Copyright (c) 2019 by The Korean College of Rheumatology. All rights reserved.

This is an Open Access article, which permits unrestricted non-commerical use, distribution, and reproduction in any medium, provided the original work is properly cited. 
To assess disease activity and outcome measures, the European League Against Rheumatism (EULAR) Sjögren's syndrome disease activity index (ESSDAI) and EULAR Sjögren's syndrome patient reported index (ESSPRI) have been developed [11,12]. The ESSDAI and ESSPRI have now been validated as outcome measures for pSS in many studies. These indices reflect 2 facets of pSS: 1) benign but disabling subjective symptoms and 2) systemic and potentially severe manifestations. The two indices reportedly had good sensitivity to change in clinical trials, although the change in the ESSPRI was smaller than that in the ESSDAI [12]. The ESSPRI levels are reportedly well-correlated with quality of life and functional status $[8,13]$. Since the two indices are poorly correlated with each other, each should be evaluated separately and independently.

However, to our knowledge, no report has investigated longitudinal ESSPRI changes in routine clinical practice for pSS. Additionally, we do not know how many patients obtain clinically meaningful ESSPRI improvement with routine therapies. We evaluated temporal changes in the ESSPRI and ESSDAI and clinical features associated with favorable ESSPRI changes in Korean patients with pSS.

\section{MATERIALS AND METHODS}

\section{Patients}

Among 104 pSS patients enrolled in a previous study [8], 63 had been followed by our rheumatology clinic and 41 were reevaluated between January 2015 and April 2016. The median follow-up period was $6.6(4.9 \sim 8.3)$ years (range 3.5 9.8 years). All patients met the 2002 American-European Consensus Group classification criteria for SS [14], and all but 1 were females. Median age was $49.0(39.0 \sim 57.0)$ years and the median duration of sicca symptoms was $8.0(3.0 \sim 10.8)$ years at enrollment. Eight (19.5\%) had moderate-to-high disease activity status and the most common extraglandular manifestation was arthralgia/arthritis (31.7\%). Baseline characteristics are summarized in Table 1. This study was approved by our institutional review board (IRB No. B-0506/ 021-004) and written informed consent was obtained from all participants.

\section{Methods}

At enrollment and follow-up, the ESSDAI, ESSPRI, and EULAR sicca score (ESS) were calculated using a previous definition $[11,15]$. Patients also completed ques- tionnaires and provided a visual analog scale (VAS) score; the short-form 36 (SF-36) and Xerostomia Inventory (XI) scores were determined, and patients were assessed for dry eye, dry mouth, joint pain, myalgia, anxiety, depression, fatigue, and global status. Demographic, clinical, and laboratory variables were collected using medical records review. Cumulative dose and prescription duration of pilocarpine, glucocorticoids, and hydroxychloroquine were calculated during the follow-up period. Satisfactory refill adherence of pilocarpine was defined as dispensed refills covering $>75 \%$ of the follow up period.

Table 1. Baseline clinical characteristics of study participants with primary Sjögren's syndrome

\begin{tabular}{|c|c|}
\hline $\begin{array}{c}\text { Demographics and } \\
\text { clinical characteristics }\end{array}$ & Total patients $(n=41)$ \\
\hline Age (yr) & $49.0(39.0 \sim 57.0)$ \\
\hline Female & $40(97.6)$ \\
\hline Menopause & $29 / 40(72.5)$ \\
\hline Duration of sicca symptoms (yr) & $8.0(3.0 \sim 10.8)$ \\
\hline Dry mouth/eye & $40(97.6) / 34(82.9)$ \\
\hline $\begin{array}{l}\text { Salivary gland*/Lacrimal gland }{ }^{\dagger} \\
\text { dysfunction }\end{array}$ & $41(100) / 28(68.3)$ \\
\hline Anti-Ro/La positivity & $38(92.7) / 20(48.8)$ \\
\hline Focal sialadenitis (focus score $\geq 1$ ) & $18 / 20(90.0)$ \\
\hline Extraglandular manifestations & $23(56.1)$ \\
\hline Arthritis/arthralgia & $13(31.7)$ \\
\hline Raynaud's phenomenon & $12(29.3)$ \\
\hline Cutaneous involvement & $6(14.6)$ \\
\hline Peripheral neuropathy & $2(4.9)$ \\
\hline Renal tubular acidosis & $1(2.4)$ \\
\hline Interstitial lung involvement & $1(2.4)$ \\
\hline \multicolumn{2}{|l|}{$\begin{array}{l}\text { Disease activity status assessed by } \\
\text { ESSDAI }\end{array}$} \\
\hline Low (ESSDAI < 5) & $33(80.5)$ \\
\hline Moderate $(5 \leq \mathrm{ESSDAI} \leq 13)$ & $7(17.1)$ \\
\hline High (ESSDAI >13) & $1(2.4)$ \\
\hline $\begin{array}{l}\text { Unsatisfactory symptom state } \\
(\text { ESSPRI } \geq 5)\end{array}$ & $16(39.0)$ \\
\hline \multicolumn{2}{|l|}{ Comorbidities } \\
\hline Hypertension & $5(12.2)$ \\
\hline Diabetes mellitus & $2(4.9)$ \\
\hline Hyperthyroidism & $2(4.9)$ \\
\hline Hypothyroidism & $8(19.5)$ \\
\hline Depression & $2(4.9)$ \\
\hline
\end{tabular}

Values are presented as median (interquartile range) or number (\%). ESSDAI: European League Against Rheumatism (EULAR) Sjögren's syndrome disease activity, ESSPRI: EULAR Sjögren's syndrome patient-reported index. *Defined using unstimulated whole salivary flow or salivary scintigraphy; ${ }^{\dagger}$ defined using Schirmer's test or Rose Bengal score. 
Based on the definition proposed by the EULAR Sjögren's Task Force [16], pSS was classified according to low (ESSDAI $<5)$, moderate $(5 \leq \mathrm{ESSDAI} \leq 13)$, and high disease activity (ESSDAI $\geq 14$ ). Additionally, unsatisfactory symptom status was defined as ESSPRI $\geq 5$, and minimal clinically important improvement was defined as an increase in ESSPRI of $\geq 1$ point or by $15 \%$, or an increase in ESSDAI of $\geq 3$ points, as previously proposed [16]. Based on temporal ESSPRI changes, pSS patients were classified into favorable (F) or unfavorable (UF) outcome subgroups. The F subgroup was defined as showing an improvement in ESSPRI (from baseline ESSPRI $\geq 5$ to follow-up ESSPRI $<5$ ) or maintenance of satisfactory symptom status (ESSPRI $<5$ ). The UF subgroup showed worsening of ESSPRI (from baseline ESSPRI $<5$ to follow-up ESSPRI $\geq 5$ ) or maintenance of unsatisfactory symptom status (ESSPRI $\geq 5$ ).

\section{Statistical analysis}

Continuous variables are expressed as median with interquartile range (25th to 75 th percentile). Statistical analysis was performed using the Mann-Whitney test for continuous variables and Wilcoxon signed-rank test for paired values. The chi-square test or Fisher's exact test was used for categorical variables. To identify clinical factors associated with favorable outcome, multivariate

Table 2. Patient-oriented measurements and laboratory variables at baseline and follow-up

\begin{tabular}{|c|c|c|c|}
\hline Variable & Baseline $(\mathrm{n}=41)$ & Follow up $(\mathrm{n}=41)$ & p-value* \\
\hline VAS for oral dryness & $70(40 \sim 85)$ & $50(24 \sim 80)$ & 0.035 \\
\hline VAS for eye dryness & $60(40 \sim 70)$ & $49(20 \sim 72)$ & 0.248 \\
\hline XI score & $42(35 \sim 47)$ & $39(29 \sim 47)$ & 0.010 \\
\hline VAS for joint pain & $10(0 \sim 49)$ & $20(5 \sim 50)$ & 0.316 \\
\hline VAS for myalgia & $20(0 \sim 49)$ & $20(0.5 \sim 40)$ & 0.604 \\
\hline VAS for anxiety & $20(0 \sim 30)$ & $10(0 \sim 40)$ & 0.641 \\
\hline VAS for depression & $20(0 \sim 40)$ & $20(0 \sim 40)$ & 0.933 \\
\hline VAS for fatigue & $50(20 \sim 60)$ & $40(20 \sim 60)$ & 0.237 \\
\hline Patient global assessment & $45(20 \sim 80)$ & $30(10 \sim 59)$ & 0.009 \\
\hline ESS & $6.33(4.67 \sim 7.67)$ & $5.00(4.67 \sim 7.33)$ & 0.182 \\
\hline ESSPRI & $4.11(3.22 \sim 5.56)$ & $5.33(3.50 \sim 6.67)$ & 0.047 \\
\hline ESSDAI & $2(1 \sim 4)$ & $2(1 \sim 4)$ & 0.936 \\
\hline \multicolumn{4}{|l|}{ SF-36 domains } \\
\hline Physical functioning & $53.91(45.84 \sim 53.91)$ & $51.61(45.85 \sim 56.22)$ & 0.679 \\
\hline Role limitation owing to physical health & $49.1(32.22 \sim 55.36)$ & $49.19(33.76 \sim 55.36)$ & 0.523 \\
\hline Bodily pain & $44.53(35.13 \sim 39.9)$ & $44.53(35.13 \sim 49.9)$ & 0.993 \\
\hline General health perceptions & $40.33(31.84 \sim 48.83)$ & $41.83(34.34 \sim 50.32)$ & 0.319 \\
\hline Vitality & $41.3(31.98 \sim 49.06)$ & $41.3(35.09 \sim 47.51)$ & 0.694 \\
\hline Social functioning & $56.14(42.18 \sim 56.14)$ & $49.16(35.2 \sim 56.14)$ & 0.159 \\
\hline Role limitations due to emotional health & $46.72(33.74 \sim 55.37)$ & $51.05(35.91 \sim 55.37)$ & 0.479 \\
\hline Physical component summary & $46.66(39.1 \sim 52.36)$ & $46.2(37.6 \sim 54.4)$ & 0.959 \\
\hline Mental component summary & $44.64(37.75 \sim 50.65)$ & $45.1(36.01 \sim 54.13)$ & 0.821 \\
\hline White blood cells $\left(/ \mathrm{mm}^{3}\right)$ & $4,880(4,020 \sim 6,030)$ & $4,930(3,920 \sim 5,790)$ & 0.620 \\
\hline Hemoglobin (g/dL) & $12.6(11.9 \sim 13.3)$ & $13.2(12.3 \sim 13.6)$ & 0.046 \\
\hline Platelet $\left(/ \mathrm{mm}^{3}\right)$ & $203(186 \sim 244)$ & $221(183 \sim 257)$ & 0.914 \\
\hline $\operatorname{ESR}(\mathrm{mm} / \mathrm{h})$ & $19(9 \sim 43)$ & $16(10 \sim 30)$ & 0.295 \\
\hline CRP (mg/dL) & $0.01(0.01 \sim 0.11)$ & $0.04(0.03 \sim 0.10)$ & 0.075 \\
\hline Total immunoglobulin G (mg/dL) & $1,980(1,710 \sim 2,460)$ & $1,710(1,530 \sim 1,950)$ & $<1.0 \times 10^{-5}$ \\
\hline Complement C3 (mg/dL) & $105.0(92.5 \sim 114.5)$ & $100.5(85.7 \sim 111.8)$ & 0.007 \\
\hline Complement C4 (mg/dL) & $19.0(15.7 \sim 23.0)$ & $22.40(18.4 \sim 24.8)$ & 0.041 \\
\hline$\beta$ 2-microglobulin & $2.16(1.81 \sim 2.68)$ & $2.25(1.73 \sim 2.67)$ & 0.975 \\
\hline
\end{tabular}

Values are presented as median (interquartile range). VAS: visual analog scale, XI: xerostomia inventory, ESS: European League Against Rheumatism (EULAR) sicca score, ESSPRI: EULAR Sjögren's syndrome patient-reported index, ESSDAI: EULAR Sjögren's syndrome disease activity, SF-36: short-form 36, ESR: erythrocyte sedimentation rate, CRP: C-reactive protein. ${ }^{*}$ Calculated using Wilcoxon signed-rank test. 
models were created using the variables with a statistical significance of $\mathrm{p}<0.1$ from the univariate analyses. Statistical analysis was performed using IBM SPSS version 21 (IBM Co., Armonk, NY, USA); $\mathrm{p}<0.05$ was considered significant.

\section{RESULTS}

\section{Longitudinal changes in patient-oriented and laboratory measurements}

The median daily dose of pilocarpine was estimated as $8.4(2.8 \sim 10.1) \mathrm{mg} /$ day in the study subjects. During the follow-up period, 17 patients (41.5\%) showed satisfactory refill adherence and 6 had never received pilocarpine. Patients with satisfactory refill adherence of pilocarpine showed a significantly higher age (55.0 [48.0 $59.0]$ vs. $43.5[36.0 \sim 53.3]$ years, $\mathrm{p}=0.011$ ) and baseline ESS $(6.33[5.33 \sim 8.50]$ vs. 5.67 [3.00 7.33], $\mathrm{p}=0.033)$ and ESSPRI (4.11 [3.17 5.95] vs. 3.78 [2.85 5.19], $\mathrm{p}=0.014$ ) levels (Table 2) than the those without satisfactory refill adherence.

After a median 6.6 years of follow-up, ESSDAI scores were not significantly changed but ESSPRI scores were statistically marginally increased $(\mathrm{p}=0.047)$. The median change was $0(-1.0 \sim 2.0)$ in ESSDAI and $0.6(-0.8 \sim$ 2.0) in ESSPRI. Six (14.6\%) patients showed clinically important improvement based on ESSDAI, as did 11 (26.8\%) based on ESSPRI, over a median of 6.6 years.

Follow-up VAS scores for dry eye or extraglandular manifestations and physical or mental component summary scores on the SF-36 were not significantly improved. However, VAS scores for dry mouth $(p=0.035)$ and XI scores $(p=0.010)$ were significantly decreased from baseline. Moreover, VAS scores for patient global assessment improved from $45(20 \sim 80)$ to $30(10 \sim 59)(\mathrm{p}=$ 0.009).

The temporal changes in ESS (from 6.33 [5.33 8.50] to 6.00 [5.00 7.17]) and ESSPRI (from 4.11 [3.17 5.95] to $5.33[3.84 \sim 6.84]$ ) were not significant in patients with satisfactory refill adherence of pilocarpine, although VAS for sicca symptoms tended to decrease (from 80 [55.0 95.0] to 47.0 [33.5 80.0] of oral dryness VAS, $p=0.062$; from 70 [45 70] to 50 [40 80] of eye dryness VAS, $\mathrm{p}=0.426$ ).

Among laboratory variables, the levels of total immunoglobulin $\mathrm{G}(\mathrm{IgG})$ were significantly decreased from $1,980(1,710 \sim 2,460)$ to $1,710(1,530 \sim 1,950) \mathrm{mg} / \mathrm{dL}$ ( $\mathrm{p}<$ $\left.1.0 \times 10^{-5}\right)$. However, the magnitude of decrease in serum
IgG levels was not significantly correlated with patient-oriented measures for sicca symptoms. Hemoglobin, C-reactive protein, and complement levels showed a statistically significant change, but these were not clinically significant.

\section{Comparison between favorable and unfavorable outcome groups}

When pSS patients were stratified into F or UF subgroups, 17 patients $(41.5 \%)$ showed a favorable outcome over a median of 6.6 years and 4 of these had unsatisfactory symptom status at baseline. In other words, 4/16 pSS patients (25.0\%) with baseline unsatisfactory symptom status achieved better symptom status during the follow-up period (Figure 1). In patients with baseline unsatisfactory symptom status, 5/16 (31.3\%) exhibited clinically meaningful improvement. However, in those with baseline satisfactory symptom status, about half $(n=12 / 25,48.0 \%)$ reported worsening of symptoms at follow-up.

When $F$ and UF outcome subgroups were compared, the F subgroup had a significantly increased prevalence of premenopausal status ( $\mathrm{p}=0.025)$ or Raynaud's phenomenon $(\mathrm{p}=0.045)$, and greater tear production (the sum of Schirmer's test results in both eyes, $\mathrm{p}=0.049$; Table 3 ). Additionally, the F subgroup had significantly less severe symptoms of dry mouth, dry eye, and depression at baseline than the UF subgroup. Accordingly, the F subgroup showed better baseline assessment using the ESS, ESSPRI, and some domains of SF-36, including bodily pain, general health, and vitality (all $\mathrm{p}<0.05$ ). At follow-up, the F outcome subgroup had a significantly lower ESSPRI $(2.67$ [2.00 4.00] vs. 6.50 [5.42 7.00], p $<1.0 \times 10^{-7}$ ) than the UF outcome subgroup. During the

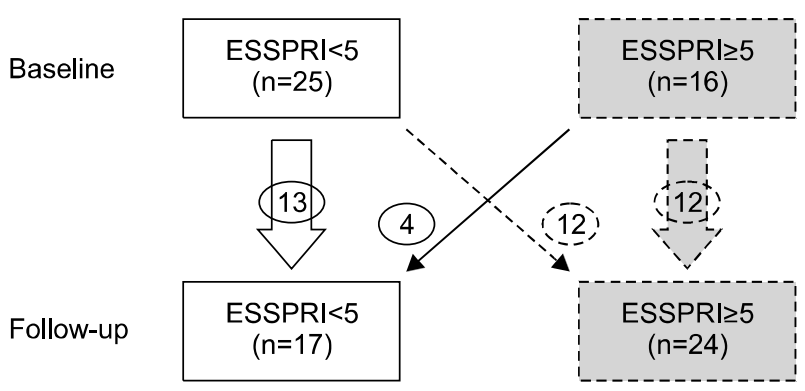

Figure 1. Flow of primary Sjögren's syndrome patients during the follow-up period based on European League Against Rheumatism Sjögren's syndrome patient reported index (ESSPRI) status. 
Table 3. Comparison between favorable (F) and unfavorable (UF) outcome subgroups*

\begin{tabular}{lccc}
\hline \hline \multicolumn{1}{c}{ Variable } & $\mathrm{F}(\mathrm{n}=17)$ & $\mathrm{UF}(\mathrm{n}=24)$ & $\mathrm{p}$-value* \\
\hline Age $(\mathrm{yr})$ & $44(40 \sim 58)$ & $51(38 \sim 58)$ & 0.156 \\
Duration of symptoms $(\mathrm{yr})$ & $7.0(3.0 \sim 10.0)$ & $9.5(2.3 \sim 13.3)$ & 0.702 \\
Menopause & $5(29.4)$ & $15 / 23(65.2)$ & 0.025 \\
Sum of Schirmer's test results $(\mathrm{mm})$ & $20(10 \sim 26)$ & $11(4 \sim 18)$ & 0.049 \\
Raynaud's phenomenon & $8(47.1)$ & $4(16.7)$ & 0.045 \\
VAS for oral dryness & $60(35 \sim 70)$ & $80(45 \sim 90)$ & 0.047 \\
VAS for eye dryness & $40(20 \sim 60)$ & $70(40 \sim 70)$ & 0.013 \\
XI score & $37(35 \sim 44)$ & $45(36 \sim 55)$ & 0.025 \\
VAS for depression & $15(0 \sim 20)$ & $20(10 \sim 40)$ & 0.043 \\
VAS for fatigue & $40(20 \sim 50)$ & $50(30 \sim 60)$ & 0.084 \\
ESS & $5.33(3.34 \sim 6.50)$ & $7.17(5.33 \sim 8.00)$ & 0.014 \\
ESSDAI & $2(1 \sim 5)$ & $2(1 \sim 3)$ & 0.182 \\
ESSPRI & $3.25(2.73 \sim 5.06)$ & $4.67(3.56 \sim 5.67)$ & 0.026 \\
ESSPRI at follow-up & $2.67(2.00 \sim 4.00)$ & $6.50(5.42 \sim 7.00)$ & $<1.0 \times 10-7$ \\
SF-36 & & & \\
Physical functioning & $53.9(49.3 \sim 53.91)$ & $51.6(44.7 \sim 56.2)$ & 0.808 \\
Role limitations due to physical health problems & $52.3(41.5 \sim 55.4)$ & $46.1(28.4 \sim 55.4)$ & 0.335 \\
Bodily pain & $45.4(42.3 \sim 53.5)$ & $40.1(32.1 \sim 45.4)$ & 0.028 \\
General health perceptions & $47.8(39.8 \sim 50.3)$ & $36.1(30.0 \sim 42.6)$ & 0.005 \\
Vitality & $47.5(41.3 \sim 50.6)$ & $35.1(28.9 \sim 43.6)$ & 0.002 \\
Social functioning & $56.1(42.2 \sim 56.1)$ & $45.7(30.0 \sim 56.1)$ & 0.085 \\
Role limitations due to emotional health problems & $46.7(40.2 \sim 55.4)$ & $44.6(29.4 \sim 55.4)$ & 0.395 \\
Physical component summary & $50.0(44.6 \sim 53.7)$ & $42.4(37.8 \sim 51.4)$ & 0.101 \\
Mental component summary & $47.8(40.8 \sim 53.7)$ & $42.5(34.3 \sim 49.0)$ & 0.106 \\
\hline Valus & &
\end{tabular}

Values are presented as median (interquartile range) or number (\%); all variables not shown in the table were not significant. VAS: visual analog scale, XI: xerostomia inventory, ESS: European League Against Rheumatism (EULAR) sicca score, ESSDAI: EULAR Sjögren's syndrome disease activity, ESSPRI: EULAR Sjögren's syndrome patient-reported index, SF-36: short-form 36. *Calculated using Mann-Whitney, chi-square or Fisher's exact test when appropriate.

follow-up period, the $\mathrm{F}$ subgroup received a significantly lower cumulative dose of pilocarpine $(\mathrm{p}=0.022)$ and glucocorticoids $(p=0.039)$, while more patients in the UF subgroup took mucolytics $(\mathrm{p}=0.004)$ and nonsteroidal anti-inflammatory drugs ( $\mathrm{p}=0.012$; Table 4$)$. Additionally, UF subgroup tended to have a higher refill adherence for pilocarpine and a higher prevalence of central nervous system acting drugs use.

We performed multivariate analysis after adjusting for covariates such as menopause, Schirmer's test results, the presence of Raynaud's phenomenon, VAS scores for oral or eye dryness, depression, and fatigue, XI scores, initial ESSPRI scores, several SF-36 scores, and medication usage. But no significant predictive factor for favorable outcome was found.

\section{DISCUSSION}

The current study is the first to investigate longitudinal changes in patient-oriented outcome measures including ESSPRI and SF-36 in Korean patients with pSS. In routine care, after a median of 6.6 years, clinically meaningful improvement in ESSPRI was observed in at most $25 \%$ of patients with pSS. Moreover, there was no significant improvement in quality-of-life measurements and subjective assessments of xerophthalmia and extraglandular symptoms, even though patient-derived measures for xerostomia and patient global assessment were significantly improved.

The number of published papers on pSS has grown exponentially in the last several decades but longitudinal patient-oriented outcomes have rarely been studied in patients with pSS. It was reported that salivary secretory function or sicca complaints in pSS were not markedly changed over a period of about 10 years $[17,18]$. In Swedish patients with pSS, symptoms of dry eye, dry mouth, and fatigue and health related quality of life did not change significantly over a period of 5 years [19]. In 
Table 4. Comparison of overall medication usage between favorable (F) and unfavorable (UF) outcome subgroups during follow-up

\begin{tabular}{|c|c|c|c|}
\hline Medication & $F(n=17)$ & UF $(n=24)$ & p-value* \\
\hline Pilocarpine use & $11(64.7)$ & $23(95.8)$ & 0.064 \\
\hline Cumulative duration (mo) & $2(0 \sim 70)$ & $47.5(33.0 \sim 66.5)$ & 0.051 \\
\hline Cumulative dose (g) & $0.14(0 \sim 14.0)$ & $13.1(0.004 \sim 23.3)$ & 0.022 \\
\hline Satisfactory refill adherence ${ }^{\dagger}$ & $5(29.4)$ & $12(50.0)$ & 0.187 \\
\hline Glucocorticoids use & $6(35.3)$ & $15(62.5)$ & 0.080 \\
\hline Cumulative duration (mo) & $0(0 \sim 9)$ & $18(0 \sim 41.7)$ & 0.021 \\
\hline Cumulative dose $(\mathrm{g})^{\mp}$ & $0(0 \sim 0.8)$ & $1.7(0 \sim 3.1)$ & 0.039 \\
\hline Hydroxychloroquine use & $12(70.6)$ & $22(91.7)$ & 0.105 \\
\hline Cumulative duration (mo) & $37(0 \sim 60)$ & $32.5(11 \sim 58)$ & 0.853 \\
\hline Cumulative dose (g) & $207.3(0 \sim 407.1)$ & $319.7(136.7 \sim 417.1)$ & 0.340 \\
\hline Mizoribine use & $4(23.5)$ & $10(41.7)$ & 0.321 \\
\hline Cumulative duration (mo) & $0(0 \sim 0)$ & $0(0 \sim 25.5)$ & 0.462 \\
\hline Cumulative dose (g) & $0(0 \sim 0)$ & $0(0 \sim 91.2)$ & 0.332 \\
\hline Mucolytic use & $3(17.6)$ & $16(66.7)$ & 0.004 \\
\hline NSAID use & $5(29.4)$ & $17(70.4)$ & 0.012 \\
\hline CNS acting xerostomic agents ${ }^{\S}$ & $3(17.6)$ & $12(50.0)$ & 0.502 \\
\hline
\end{tabular}

Values are presented as number (\%) or median (interquartile range). NSAID: nonsteroidal anti-inflammatory drug, CNS: central nervous system. * Calculated using Mann-Whitney, chi-square, or Fisher's exact test when appropriate. ${ }^{\dagger}$ Dispensed refills covering $>$ $75 \%$ of the follow up period of each patient; ${ }^{\dagger}$ calculated by adding prednisolone equivalents; ${ }^{{ }}$included tricyclic agents, selective serotonin reuptake inhibitors, serotonin and norepinephrine reuptake inhibitors, benzodiazepines, trazodone, and zolpidem.

another longitudinal study on temporal changes, self-reported fatigue levels were unchanged over a period of 5 years in Norwegian pSS patients [20]. Moreover, a recent study using salivary gland ultrasound outcomes revealed that abnormalities did not change significantly during a follow-up of nearly 2 years [21].

The current study also found no clinically meaningful changes in most patients with pSS; $85 \%$ and $73 \%$ did not experience clinically important improvement in ESSDAI and ESSPRI, respectively. Such stability of patient-oriented outcome measures or symptoms suggests that the current treatment modalities provide only very limited efficacy. In the current study, ESSPRI was not significantly reduced even in subjects with satisfactory refill adherence of pilocarpine although there was a tendency to decrease in VAS for sicca symptoms. It has been well established that pSS patients have significantly impaired quality of life and this may produce a floor effect. However, unlike the abovementioned studies, our participants reported significant improvement in xerostomia and global assessment of SS during the follow-up period. This can be explained by the finding that more patients $(34 / 41,82.9 \%)$ had taken cholinergics in our study when compared to previous studies $(2 / 58$ [4.4\%] in the Swedish study) [19]. Moreover, diagnosis and education during regular visits can reduce the emotional impact of anxiety and depression, although mental component scores in the SF-36 were not improved.

Concerning laboratory parameters, we observed that only serum IgG levels were significantly decreased during the follow-up period. Theander et al. [19] previously reported that serum IgG levels were not changed over a period of 5 years, but Pertovaara et al. [18] reported that levels significantly decreased after a median of 9 years. Several treatments including abatacept and hydroxychloroquine reportedly decreased the serum IgG levels in pSS patients [22-24]. In the current study, 34 (82.9\%) of 41 study subjects had taken hydroxychloroquine and 21 (51.2\%) had received glucocorticoids during follow-up. These treatments could reduce serum IgG levels. However, the decrease in serum IgG levels was not associated with subjective improvement of xerostomia in our study.

Although pSS patients have a higher risk of nonHodgkin lymphoma (NHL) than controls or patients with systemic lupus erythematosus, most have relatively chronic stable disease without rapid deterioration of exocrine function or dramatic changes in extra-exocrine clinical features [25-27]. Nevertheless, many researchers have focused only on the risk factors for NHL that develop in a small proportion of pSS patients $(<2 \%)[28,29]$. Until now, no study has investigated predictive factors for patient-oriented outcomes or longitudinal change in the 
prevalent symptoms. In this context, we divided our pSS patients into favorable and unfavorable subgroups and compared clinical features between the two subgroups.

In the current study, about $40 \%$ showed a favorable course and even about $30 \%$ of pSS patients with baseline ESSPRI $>5$ achieved clinically meaningful improvement, although the $\mathrm{F}$ subgroup used less medication and tended to have low prevalence of satisfactory refill adherence of pilocarpine than the UF subgroup. Because of small sample size, multivariate analysis did not demonstrate an independent factor associated with favorable outcomes. However, in univariate analyses, better patient assessment of sicca symptoms and depression was associated with favorable outcomes. Therefore, the psychological attitude toward the disease or the background psychological profile may be an underlying factor associated with favorable outcomes, because ESSPRI is correlated with the mental health subdimension of SF-36 or depression $[8,30]$.

Premenopausal status, the presence of extraglandular manifestations such as Raynaud's phenomenon, or residual lacrimal gland function at diagnosis was also associated with a favorable course. The prevalence of Raynaud's phenomenon was reported to be $16 \% \sim 35 \%$ in a large case series of pSS patients, and Raynaud's phenomenon preceded the onset of sicca symptom in about half of pSS patients $[31,32]$. Several studies showed that the age at disease onset (defined as the first subjective experience of glandular or extraglandular symptoms) does not influence clinical features $[33,34]$. In the current study, disease duration was comparable between F and UF outcome subgroups. However, in a large Spanish cohort study, younger-onset pSS showed a lower prevalence of xerostomia and abnormal ocular findings [31]. Therefore, when considering the abovementioned findings together, a higher prevalence of premenopausal women in the F subgroup may suggest that early diagnosis without severe exocrine impairment could be associated with a favorable outcome in pSS. Unfortunately, we know little about the natural history of pSS as well as the transition from the preclinical phase into clinical disease. pSS-related autoantibodies can be detected at a median of $4 \sim 5$ years before clinical diagnosis. In addition, immune-meditated glandular injury is considered to progress over several years and the delay between symptom onset and final diagnosis has been reported to range from $3 \sim 10$ years $[35,36]$. Therefore, when the diagnosis of pSS is made after the development of sicca symptoms, the patient may already have irreversible exocrine dysfunction. Moreover, delayed diagnosis could increase the psychological distress caused by unexplained symptoms in pSS patients [10]. Thus, diagnosis in the state with lower scores on sicca symptoms or with residual exocrine function could be important to improve patient-oriented outcomes and to help decrease the local or systemic complications.

Our study has several limitations. First, the rate of loss to follow-up was high (about 60\%). Those without symptomatic improvement were more likely to be lost to follow-up and the proportion of patients achieving clinically meaningful improvement could be overestimated in this study. However, we believe that the importance of early diagnosis in pSS is not overemphasized. Second, the sample size was inadequate for multivariate analysis. Hence, a larger number of patients with pSS is needed for study. Third, most patients had low-to-moderate disease activity and did not have severe systemic manifestations. However, the ESSDAI level (median 2.0) in our patients was similar to that in the French Assessment of Systemic Signs and Evolution in Sjögren's syndrome (ASSESS) cohort and in patients enrolled in a randomized clinical trial of hydroxychloroquine (the JOQUER trial) [37]. Therefore, our study subjects reflected general rather than unique features of pSS patients.

\section{CONCLUSION}

In summary, our study showed that $27 \%$ showed clinically important improvement in ESSPRI over a median of 6.6 years and $40 \%$ showed a favorable course based on ESSPRI in routine care. Favorable patient-derived outcome was associated with some clinical features, including premenopausal status, greater lacrimal flow, and fewer sicca or depressive symptoms at baseline.

\section{CONFLICT OF INTEREST}

No potential conflict of interest relevant to this article was reported.

\section{REFERENCES}

1. Rischmueller M, Tieu J, Lester S. Primary Sjögren's syndrome. Best Pract Res Clin Rheumatol 2016;30:189-220.

2. Brito-Zerón P, Baldini C, Bootsma H, Bowman SJ, Jonsson $\mathrm{R}$, Mariette X, et al. Sjögren syndrome. Nat Rev Dis Primers 2016;2:16047. 
3. Cornec D, Chiche L. Is primary Sjögren's syndrome an orphan disease? A critical appraisal of prevalence studies in Europe. Ann Rheum Dis 2015;74:e25.

4. Qin B, Wang J, Yang Z, Yang M, Ma N, Huang F, et al. Epidemiology of primary Sjögren's syndrome: a systematic review and meta-analysis. Ann Rheum Dis 2015;74:1983-9.

5. Flores-Chávez A, Kostov B, Solans R, Fraile G, Maure B, Feijoo-Massó C, et al.; GEAS-SS SEMI Registry. Severe, life-threatening phenotype of primary Sjögren's syndrome: clinical characterisation and outcomes in 1580 patients (GEAS-SS Registry). Clin Exp Rheumatol 2018;36 Suppl 112:121-9.

6. Theander E, Manthorpe R, Jacobsson LT. Mortality and causes of death in primary Sjögren's syndrome: a prospective cohort study. Arthritis Rheum 2004;50:1262-9.

7. Singh AG, Singh S, Matteson EL. Rate, risk factors and causes of mortality in patients with Sjögren's syndrome: a systematic review and meta-analysis of cohort studies. Rheumatology (Oxford) 2016;55:450-60.

8. Cho HJ, Yoo JJ, Yun CY, Kang EH, Lee HJ, Hyon JY, et al. The EULAR Sjogren's syndrome patient reported index as an independent determinant of health-related quality of life in primary Sjogren's syndrome patients: in comparison with non-Sjogren's sicca patients. Rheumatology (Oxford) 2013;52:2208-17.

9. Strömbeck B, Ekdahl C, Manthorpe R, Wikström I, Jacobsson L. Health-related quality of life in primary Sjögren's syndrome, rheumatoid arthritis and fibromyalgia compared to normal population data using SF-36. Scand J Rheumatol 2000;29:20-8.

10. Segal B, Bowman SJ, Fox PC, Vivino FB, Murukutla N, Brodscholl J, et al. Primary Sjögren's syndrome: health experiences and predictors of health quality among patients in the United States. Health Qual Life Outcomes 2009;7:46.

11. Seror R, Ravaud P, Mariette X, Bootsma H, Theander E, Hansen A, et al.; EULAR Sjögren's Task Force. EULAR Sjogren's Syndrome Patient Reported Index (ESSPRI): development of a consensus patient index for primary Sjogren's syndrome. Ann Rheum Dis 2011;70:968-72.

12. Seror R, Theander E, Bootsma H, Bowman SJ, Tzioufas A, Gottenberg JE, et al. Outcome measures for primary Sjögren's syndrome: a comprehensive review. J Autoimmun 2014;51:51-6.

13. Hackett KL, Newton JL, Frith J, Elliott C, Lendrem D, Foggo $\mathrm{H}$, et al. Impaired functional status in primary Sjögren's syndrome. Arthritis Care Res (Hoboken) 2012;64:1760-4.

14. Vitali C, Bombardieri S, Jonsson R, Moutsopoulos HM, Alexander EL, Carsons SE, et al.; European Study Group on Classification Criteria for Sjögren's Syndrome. Classification criteria for Sjögren's syndrome: a revised version of the European criteria proposed by the American-European Consensus Group. Ann Rheum Dis 2002;61:554-8.

15. Seror R, Bowman SJ, Brito-Zeron P, Theander E, Bootsma $\mathrm{H}$, Tzioufas A, et al. EULAR Sjögren's syndrome disease activity index (ESSDAI): a user guide. RMD Open 2015;1: e000022.

16. Seror R, Bootsma H, Saraux A, Bowman SJ, Theander E, Brun JG, et al.; EULAR Sjögren's Task Force. Defining disease activity states and clinically meaningful improvement in primary Sjögren's syndrome with EULAR primary Sjögren's syndrome disease activity (ESSDAI) and pa- tient-reported indexes (ESSPRI). Ann Rheum Dis 2016; 75:382-9.

17. Gannot G, Lancaster HE, Fox PC. Clinical course of primary Sjögren's syndrome: salivary, oral, and serologic aspects. J Rheumatol 2000;27:1905-9.

18. Pertovaara M, Pukkala E, Laippala P, Miettinen A, Pasternack A. A longitudinal cohort study of Finnish patients with primary Sjögren's syndrome: clinical, immunological, and epidemiological aspects. Ann Rheum Dis 2001;60:467-72.

19. Theander E, Andersson SI, Manthorpe R, Jacobsson LT. Proposed core set of outcome measures in patients with primary Sjögren's syndrome: 5 year follow up. J Rheumatol 2005;32:1495-502.

20. Haldorsen K, Bjelland I, Bolstad AI, Jonsson R, Brun JG. A five-year prospective study of fatigue in primary Sjögren's syndrome. Arthritis Res Ther 2011;13:R167.

21. Gazeau P, Cornec D, Jousse-Joulin S, Guellec D, Saraux A, Devauchelle-Pensec V. Time-course of ultrasound abnormalities of major salivary glands in suspected Sjögren's syndrome. Joint Bone Spine 2018;85:227-32.

22. Kruize AA, Hené RJ, Kallenberg CG, van Bijsterveld OP, van der Heide A, Kater L, et al. Hydroxychloroquine treatment for primary Sjögren's syndrome: a two year double blind crossover trial. Ann Rheum Dis 1993;52:360-4.

23. Miyawaki S, Nishiyama S, Matoba K. Efficacy of low-dose prednisolone maintenance for saliva production and serological abnormalities in patients with primary Sjögren's syndrome. Intern Med 1999;38:938-43.

24. Nakayamada S, Saito K, Nakatsuka K, Nakano K, Tokunaga $\mathrm{M}$, Sawamukai N, et al. Efficacy of mizoribine treatment in patients with Sjögren's syndrome: an open pilot trial. Mod Rheumatol 2003;13:339-45.

25. Liang Y, Yang Z, Qin B, Zhong R. Primary Sjogren's syndrome and malignancy risk: a systematic review and meta-analysis. Ann Rheum Dis 2014;73:1151-6.

26. Rúa-Figueroa I, Fernández Castro M, Andreu JL, SanchezPiedra C, Martínez-Taboada V, Olivé A, et al.; Sjogrenser and Relesser Researchers and EAS-SER Group. Comorbidities in patients with primary Sjögren's syndrome and systemic lupus erythematosus: a comparative registries-based study. Arthritis Care Res (Hoboken) 2017;69:38-45.

27. Haldorsen K, Moen K, Jacobsen H, Jonsson R, Brun JG. Exocrine function in primary Sjögren syndrome: natural course and prognostic factors. Ann Rheum Dis 2008;67: 949-54.

28. Johnsen SJ, Brun JG, Gøransson LG, Småstuen MC, Johannesen TB, Haldorsen K, et al. Risk of non-Hodgkin's lymphoma in primary Sjögren's syndrome: a populationbased study. Arthritis Care Res (Hoboken) 2013;65:816-21.

29. Nishishinya MB, Pereda CA, Muñoz-Fernández S, PegoReigosa JM, Rúa-Figueroa I, Andreu JL, et al. Identification of lymphoma predictors in patients with primary Sjögren's syndrome: a systematic literature review and meta-analysis. Rheumatol Int 2015;35:17-26.

30. Omma A, Tecer D, Kucuksahin O, Sandikci SC, Yildiz F, Erten S. Do the European League Against Rheumatism (EULAR) Sjögren's syndrome outcome measures correlate with impaired quality of life, fatigue, anxiety and depression in primary Sjögren's syndrome? Arch Med Sci 2018;14: 830-7.

31. Ramos-Casals M, Solans R, Rosas J, Camps MT, Gil A, Del 
Pino-Montes J, et al.; GEMESS Study Group. Primary Sjögren syndrome in Spain: clinical and immunologic expression in 1010 patients. Medicine (Baltimore) 2008;87: 210-9.

32. García-Carrasco M, Sisó A, Ramos-Casals M, Rosas J, de la Red G, Gil V, et al. Raynaud's phenomenon in primary Sjögren's syndrome. Prevalence and clinical characteristics in a series of 320 patients. J Rheumatol 2002;29:726-30.

33. Tishler M, Yaron I, Shirazi I, Yaron M. Clinical and immunological characteristics of elderly onset Sjögren's syndrome: a comparison with younger onset disease. J Rheumatol 2001;28:795-7.

34. Botsios C, Furlan A, Ostuni P, Sfriso P, Andretta M, Ometto F, et al. Elderly onset of primary Sjögren's syndrome: clinical manifestations, serological features and oral/ocular diagnostic tests. Comparison with adult and young onset of the disease in a cohort of 336 Italian patients. Joint Bone
Spine 2011;78:171-4.

35. Mignogna MD, Fedele S, Lo Russo L, Lo Muzio L, Wolff A. Sjögren's syndrome: the diagnostic potential of early oral manifestations preceding hyposalivation/xerostomia. J Oral Pathol Med 2005;34:1-6.

36. Theander E, Jonsson R, Sjöström B, Brokstad K, Olsson P, Henriksson G. Prediction of Sjögren's syndrome years before diagnosis and identification of patients with early onset and severe disease course by autoantibody profiling. Arthritis Rheumatol 2015;67:2427-36.

37. Gottenberg JE, Seror R, Miceli-Richard C, Benessiano J, Devauchelle-Pensec V, Dieude P, et al. Serum levels of beta2-microglobulin and free light chains of immunoglobulins are associated with systemic disease activity in primary Sjögren's syndrome. Data at enrollment in the prospective ASSESS cohort. PLoS One 2013;8:e59868. 